Skin

Appendage

Disorders
Skin Appendage Disord 2018;4:354-357

DOI: $10.1159 / 000485569$
Received: September 1, 2017

Accepted: November 22, 2017

Published online: December 21, 2017

\title{
Chronic Crusted Scalp Lesion of an Elderly Male
}

\author{
A. Caresse Gamret ${ }^{a}$ Raymond M. Fertig ${ }^{a} \quad$ Evan Darwin $^{a} \quad$ Kyle Klingbeil $^{a}$ \\ Eugene Sanik $^{\mathrm{b}}$ Sudeep Gaudic \\ a University of Miami, Miller School of Medicine, Department of Dermatology and Cutaneous Surgery, Miami, FL, USA; \\ b James A. Haley VA Hospital, Tampa, FL, USA; ' $P$ Pathology and Laboratory Medicine Service, James A. Haley VA Hospital, \\ Tampa, FL, USA
}

\section{Question}

A 75-year-old man presented to clinic with a $4.5 \times 2.5$ $\mathrm{cm}$ crusted lesion of his front left scalp in an area of androgenic alopecia. This nonhealing lesion developed 6 months prior following cryotherapy of actinic keratosis of the scalp. Physical exam revealed an erythematous skin erosion with thick crusts (Fig. 1). After removal of the crust, pustules were present. Previous treatment with topical and systemic antibiotics was not successful in resolving the lesion. The pustules with crusting overlying erod- ed nodules were accompanied by a surrounding region of further hair loss. The man reported that the scalp lesion was not painful, nor did it itch. A skin biopsy of the region was performed and revealed parakeratotic epidermis with lymphocytes and an inflammatory plasma cell infiltrate (Fig. 2, Fig. 3). Mycobacterial, viral, and fungal cultures of the scalp lesion were negative. Laboratory values such as blood cell counts, liver transaminases, blood urea nitrogen, and serum creatinine levels were within normal ranges.

What is your diagnosis?

\section{KARGER}

(c) 2017 S. Karger AG, Basel

E-Mail karger@karger.com

www.karger.com/sad
A. Caresse Gamret

University of Miami, Miller School of Medicine

Department of Dermatology and Cutaneous Surgery

1475 NW 12th Ave, 2nd Floor, Miami, FL 33136 (USA)

E-Mail acgamret@gmail.com 


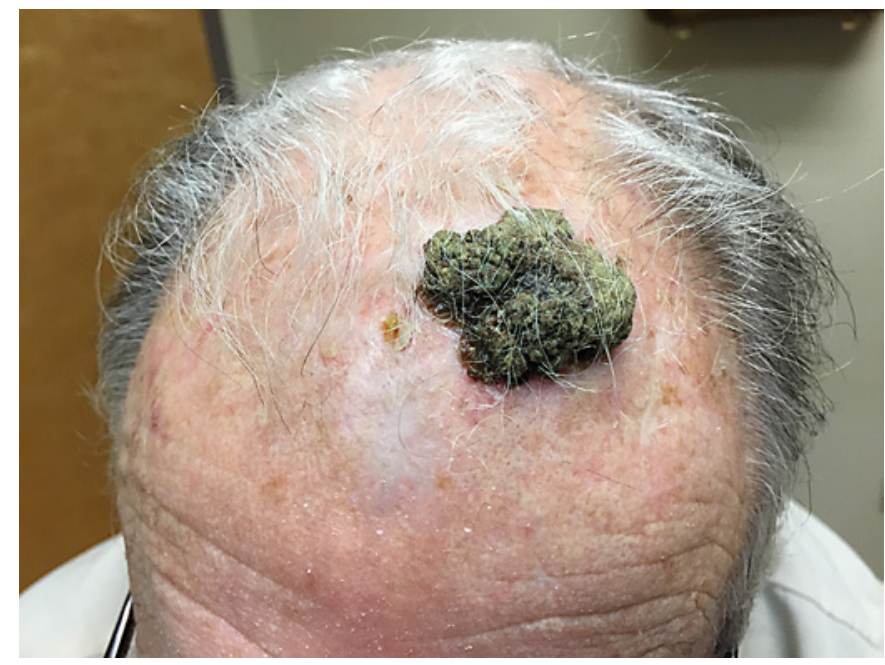

Fig. 1. Initial presentation showing the crusted lesion on the scalp.

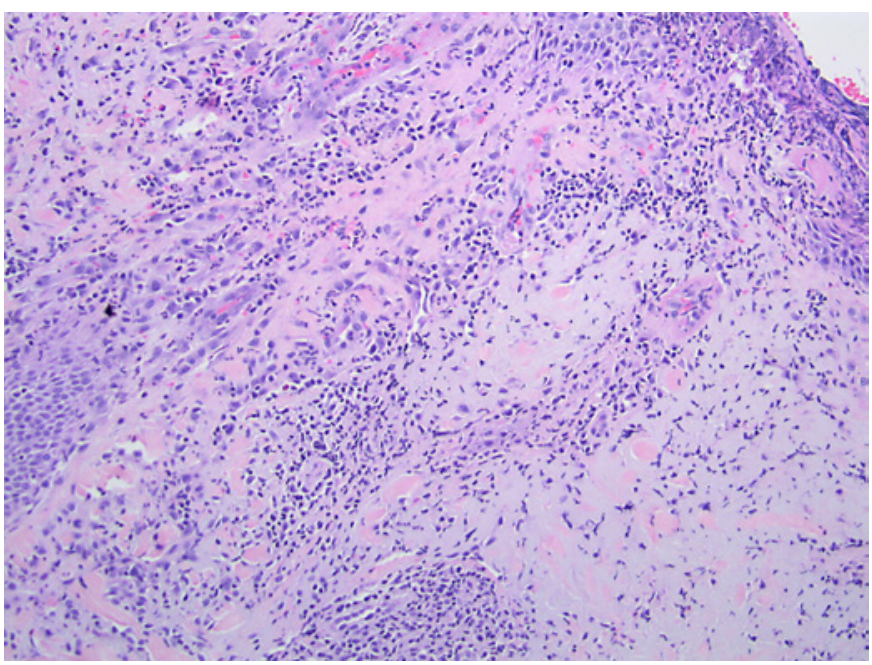

Fig. 3. Biopsy photo showing lymphocyte and plasma cell infiltrate (hematoxylin \& eosin stain, original magnification $\times 20$ ).

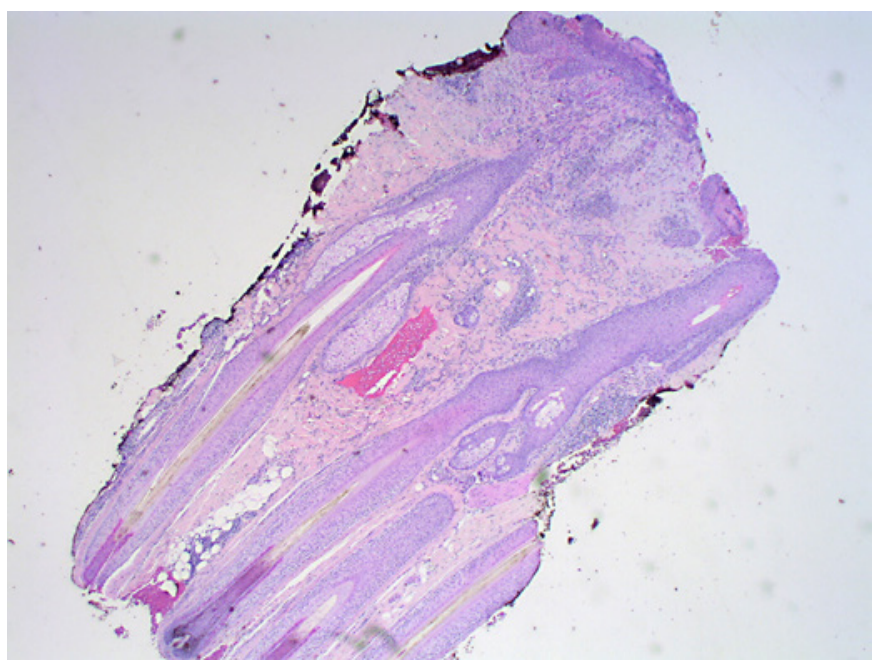

Fig. 2. Biopsy photo showing lymphocyte and plasma cell infiltrate (hematoxylin \& eosin stain, original magnification $\times 2$ ). 


\section{Answer}

Erosive pustular dermatosis of the scalp

Erosive pustular dermatosis of the scalp (EPDS) is a rare inflammatory condition of the skin. It typically plagues the elderly, primarily women (women to men 2: 1), following trauma to the scalp or longstanding sun damage, and is infrequently seen in children $[1,2]$. Recurrent erosions and yellow to brown crusts and pustules over time result in scarring and subsequent alopecia, telangiectasia, and atrophy of the skin [2]. The hyperkeratotic lesions are not painful and pruritus is rare [3]. Triggers have been reported to include imiquimod [4], ingenol mebutate [5], and herpes zoster [6]. Corticosteroids have been proven to be effective in the treatment of most of these cases.

The pathology of EPDS is not fully understood but it has been proposed to be due to atrophy of skin tissues and is marked by the presence of nonspecific inflammation with an abundance of neutrophils [7]. The response to corticosteroids by EPDS suggests immunological dysfunction may be related to the pathomechanism, and the response to corticosteroids can aid in diagnosis [7]. Among the most common causes of EPDS are lacerations, bruises, shingles, severe sunburns that result in blistering, surgical resections, skin grafting, topical chemotherapy, and cryotherapy, yet EPDS persists long after the initial insult [3]. Autoimmune diseases also have been reported in association with EPDS such as rheumatoid arthritis [7]. Because the clinical appearance of EPDS presents very similar to bacterial or fungal infections such as a kerion, in most instances colonizing organisms are misinterpreted as the cause of the disease leading to inappropriate antibiotic and antifungal treatments that fail to resolve the lesion, as the condition is not primarily an infective process $[1,7]$.

In the case of our patient, a history of cryotherapy and lymphocytes and plasma cells noted upon biopsy were the basis of the diagnosis of EPDS. The lesion was treated with high-potency topical corticosteroid ointment (clobetasol $0.05 \%$ ) applied daily. This treatment regimen resulted in the lesion clearing in 1 month.

Among disorders to be considered in the differential diagnosis for EPDS would be tinea capitis and kerion celsi, in which a mycological investigation would be sufficient to differentiate; actinic keratosis and squamous cell carcinoma should also be considered, which are distinguishable by biopsy. Subcorneal pustular dermatosis should be ruled out, which is more closely related to EPDS but has blisters and hypopyon and is not limited to the scalp. Finally, the autoimmune disease pemphigus foliaceus can be ruled out with a Tzanck smear test [3].

In instances when topical corticosteroids fail to resolve the lesions, which is thought to be due to the inability to penetrate hyperkeratosis crusts, a new treatment option has been discovered: curettage followed by aminolevulinic acid photodynamic therapy (PDT) [8]. Curettage is used to promote reepithelization within a week and the following treatment is characterized by a 1-hour application of $20 \%$ aminolevulinic acid and blue light $(417 \mathrm{~nm})$ exposure for 16 min 1-2 weeks later [8]. In the 8 patients this treatment was assessed in, the lesions cleared after one round of treatment; in the other 2, two rounds successfully cleared the EPDS. PDT is also thought to be effective in combination with topical corticosteroids for recurrent lesions, as PDT may increase the responsiveness to anti-inflammatory agents [8].

\section{Statement of Ethics}

This study complies with guidelines for human studies and animal welfare regulations. Subjects have given their informed consent and the protocol was approved by the institute's committee on human research. While this study did not include animal experiments it conforms to all institutional standards.

\section{Disclosure Statement}

The authors have no conflicts of interest to report.

\section{Keywords}

Erosive pustular dermatosis · Cryotherapy $\cdot$ Scalp dermatoses
Gamret/Fertig/Darwin/Klingbeil/Sanik/ Gaudi 


\section{References}

1 Grattan CEH, Peachey RD, Boon A: Evidence for a role of local trauma in the pathogenesis of erosive pustular dermatosis of the scalp. Clin Exp Dermatol 1988;13:7-10.

-2 Shimada R, Masu T, Hanamizu H, Aiba S, Okuyama R: Infantile erosive pustular dermatosis of the scalp associated with Klippel-Feil syndrome. Acta Derm Venereol 2010;90: 200-201.

3 Semkova K, Tchernev G, Wollina U: Erosive pustular dermatosis (chronic atrophic dermatosis of the scalp and extremities). Clin Cosmet Investig Dermatol 2013;6:177-182.
4 Corradin MT, Forcione M, Giulioni E, Fiorentino R, Ferrazzi A, Alaibac M: Erosive pustular dermatosis of the scalp induced by imiquimod. Case Rep Dermatol Med 2012;2012: 828749.

5 Rongioletti F, Chinazzo C, Javor S: Erosive pustular dermatosis of the scalp induced by ingenol mebutate. J Eur Acad Dermatol Venereol 2016;30:e110-e111.

6 Kim KR, Lee JY, Kim MK, Yoon TY: Erosive pustular dermatosis of the scalp following herpes zoster: successful treatment with topical tacrolimus. Ann Dermatol 2010;22:232234.
Patton D, Lynch PJ, Fung MA, Fazel N: Chronic atrophic erosive dermatosis of the scalp and extremities: a recharacterization of erosive pustular dermatosis. J Am Acad Dermatol 2007;57:421-427.

8 Yang CS, Kuhn H, Cohen LM, Kroumpouzos G: Aminolevulinic acid photodynamic therapy in the treatment of erosive pustular dermatosis of the scalp: a case series. JAMA Dermatol 2016;152:694-697. 